Macmillan Professional Masters

Landlord and Tenant Law 
Macmillan Professional Masters

Law titles Law Series Editor Marise Cremona

Basic English Law W. T. Major

Company Law Janet Dine

Constitutional and Administrative Law John Alder

Contract Law Ewan McKendrick

Conveyancing Priscilla Sarton

Criminal Law Marise Cremona

Employment Law Debbie Lockton

European Community Law Josephine Shaw

Family Law Kate Standley

Land Law Kate Green

Landlord and Tenant Law Margaret Wilkie and Godfrey Cole

Law of Trusts Patrick McLoughlin and Catherine Rendell

Legal Method Ian McLeod

Torts Alastair Mullis and Ken Oliphant

\section{Business Titles}

Communication Nicki Stanton

Company Accounts Roger Oldcorn

Cost and Management Accounting Roger Hussey

Data Processing John Bingham

Employee Relations Chris Brewster

Financial Management Geoffrey Knott

Management Roger Oldcorn

Marketing Robert G. I. Maxwell

Marketing for the Non-Profit Sector Tim Hannagan

Marketing Research John Frain

Office Administration E. C. Eyre

Operations Management Howard Barnett

Personnel Management Margaret Attwood

Study Skills Kate Williams

Supervision Mike Savedra and John Hawthorn

Systems Analysis John Bingham and Garth Davies 


\section{Landlord and Tenant Law}

Second edition

Margaret Wilkie, $L L M$

Solicitor, Principal Lecturer in Law, Westminster University

and

Godfrey Cole, $L L M$

Barrister, Full-time Chair of Tribunals, Independent Tribunal Service

Law Series Editor: Marise Cremona

Senior Fellow, Centre for Commercial Law Studies, Queen Mary

Westfield College, University of London 
(C) Margaret Wilkie and Godfrey Cole 1989, 1993

All rights reserved. No reproduction, copy or transmission of this publication may be made without written permission.

No paragraph of this publication may be reproduced, copied or transmitted save with written permission or in accordance with the provisions of the Copyright, Designs and Patents Act 1988, or under the terms of any licence permitting limited copying issued by the Copyright Licensing Agency, 90 Tottenham Court Road, London W1P 9HE.

Any person who does any unauthorised act in relation to this publication may be liable to criminal prosecution and civil claims for damages.

First edition 1989

Reprinted 1992

Second edition 1993

Published by

THE MACMILLAN PRESS LTD

Houndmills, Basingstoke, Hampshire RG21 2XS

and London

Companies and representatives

throughout the world

ISBN 978-0-333-58911-3

ISBN 978-1-349-13166-2 (eBook)

DOI 10.1007/978-1-349-13166-2

A catalogue record for this book is available from the British Library.

$\begin{array}{rrrrrrrrrr}10 & 9 & 8 & 7 & 6 & 5 & 4 & 3 & 2 & 1\end{array}$

$\begin{array}{llllllllll}02 & 01 & 00 & 99 & 98 & 97 & 96 & 95 & 94 & 93\end{array}$ 


\section{Contents}

Table of Cases

Table of Statutes

$\mathbf{x}$

Preface

xix

xxvii

Part I LeASES: THE GENERAL LAW

1 Essential characteristics of a lease 3

1.1 An estate in land 3

1.2 Fixed duration 4

1.3 Exclusive possession of land 4

1.4 Rent 5

1.5 Reversion expectant upon the term 5

1.6 Distinction between a lease and a licence 6 Case notes $\quad 8$

Summary 9

Exercises $\quad 10$

$\begin{array}{ll}\text { Workshop } & 10\end{array}$

2 Different types of leases 11

2.1 Tenancy for a fixed term 11

2.2 Periodic tenancies 11

$\begin{array}{lll}2.3 & \text { Statutory tenancies } & 12\end{array}$

2.4 Tenancies terminable on death or marriage $\quad 12$

2.5 Perpetually renewable leases 13

2.6 Reversionary leases 13

2.7 Concurrent leases 14

2.8 Tenancies by estoppel 14

2.9 Tenancies at will 14

2.10 Tenancies at sufferance $\quad 15$

Case notes $\quad 15$

Summary $\quad 16$

Exercises $\quad 17$

$\begin{array}{ll}\text { Workshop } & 17\end{array}$ 
3 Formalities for creating a lease

3.1 Contractual nature of a lease creating an estate in land

3.2 Requirements for a legal lease 20

3.3 Leases enforceable in equity: enforceable contracts 20

3.4 Leases enforceable in equity: part performance and other equitable principles

3.5 The nature and effect of an equitable lease 21 Case notes

Summary

Exercises

Workshop

4 Covenants and conditions in leases

4.1 Difference between conditions and covenants 25

4.2 Covenants in leases 25

Case note $\quad 27$

Summary $\quad 27$

Exercises $\quad 27$

5 Obligations implied on behalf of the landlord 28

5.1 Landlord's covenant for quiet enjoyment 28

5.2 Landlord's covenant not to derogate from his grant 29

5.3 Implied obligations as to the state of fitness of the premises

Case and statute notes $\quad 34$

Summary $\quad 39$

Exercises $\quad 40$

Workshop $\quad 40$

6 Obligations implied on behalf of the tenant 41

6.1 To pay rent 41

6.2 To pay rates and charges payable by the occupier of the premises

6.3 Tenant's implied obligations as to maintenance and repair

6.4 Obligation to allow landlord to enter and view state of repair of the premises

Notes

Summary

Exercises

Workshop 
7 Enforceability of covenants between successors in title to the landlord and tenant

7.1 Privity of contract

7.2 Difference between an assignment and a sublease

7.3 Enforceability of covenants on an assignment of a lease

7.4 Indemnity on assignment

7.5 Assignment of the reversion

7.6 Position on assignment of an equitable lease or its reversion

7.7 Enforceability of covenants against a sublessee $\quad 50$

Case notes $\quad 51$

Summary $\quad 52$

Exercises $\quad 53$

Workshop 54

8 The effects of some covenants often included in leases 55

8.1 Repairing covenants 55

8.2 Reservation of rent 65

8.3 Covenants against assignment or underletting 73

8.4 Covenants restrictive of the user of the premises 78

8.5 Covenants against alterations or improvements $\quad 80$

8.6 Covenant to insure the premises 81

8.7 Options to renew the lease, break clauses and options to purchase the reversion $\quad 82$

Case and statute notes $\quad 84$

Summary $\quad 91$

Exercises $\quad 94$

Workshop 96

9 Enforceability of a lease against a purchaser of the freehold reversion: title to leasehold property 98

9.1 Registered and unregistered title 98

9.2 Unregistered title: enforceability of a lease 98

9.3 Registered title: enforceability of a lease 99

9.4 Title to leasehold property: unregistered land 101

9.5 Title to leasehold property: registered land 101

9.6 Short form of model lease 102

Case and statute notes 105

Summary 105

Exercises $\quad 106$

$\begin{array}{ll}\text { Workshop } & 106\end{array}$ 
10 Termination of a lease 107

10.1 Effluxion of time 107

$\begin{array}{ll}10.2 \text { Notice to quit } & 107\end{array}$

10.3 Surrender 108

10.4 Merger 109

10.5 Forfeiture 109

10.6 Disclaimer 113

10.7 Frustration 113

10.8 Repudiating breach 113

10.9 Tenant's fixtures 113

Case and statute notes $\quad 114$

$\begin{array}{ll}\text { Summary } & 117\end{array}$

Exercises $\quad 118$

Workshop 118

Part II LEASES: STATUTORY LAW 122

11 Private residential accommodation 123

11.1 Introduction 123

11.2 Concepts applicable to all residential tenancies $\quad 126$

11.3 Excluded lettings 131

11.4 The transitional provisions 134

11.5 Rent Act lettings 135

11.6 Assured tenancies $\quad 140$

11.7 Other forms of protection 145

Annex 1 Possession, Rent Act $1977 \quad 150$

Annex 2 Possession, Housing Act 1988

Annex 3 Suitable Alternative Accommodation $\quad 157$

Summary 158

Exercises $\quad 159$

Workshop 160

12 Private residential accommodation: long leases 162

12.1 Introduction 162

12.2 Control of service charges 163

12.3 Landlord \& Tenant Act 1987

12.4 Landlord \& Tenant Act 1954 Part I 167

12.5 Leasehold Reform Act 1967

12.6 Leasehold Reform, Housing and Urban
Development Act 1993

Summary 174

$\begin{array}{ll}\text { Exercises } & 175\end{array}$ 
13 Public sector residential accommodation 176

13.1 Introduction and scope 176

13.2 Secure tenancies under the Housing Act 1985

Summary 188

Exercises $\quad 189$

$\begin{array}{ll}\text { Workshop } & 189\end{array}$

14 Business tenancies 191

14.1 Introduction 191

14.2 The right of renewal 194

14.3 The position until renewal 198

14.4 The renewal procedure $\quad 198$

14.5 A new tenancy 201

14.6 The grounds of opposition 203

14.7 Compensation at the end of a tenancy 207

Summary 209

Exercises $\quad 210$

Workshop 211

15 Agricultural holdings 212

15.1 Introduction 212

15.2 Scope of the legislation 213

15.3 The terms of an agricultural lease 214

15.4 Security of tenure $\quad 215$

15.5 Compensation 218

15.6 Other interests $\quad 219$

15.7 Agricultural tied homes 220

Summary 221

Exercises $\quad 221$

Further Reading 222

Index

223 


\section{Table of Cases}

Abbey National Building Society $v$. Maybeech [1984] 3 All ER 262 $73,111,112$

Abernethie v. A. M. \& J. Kleinman [1969] 2 WLR 1364 Adams $v$. Green [1978] 247 EG $49 \quad 202$ Addiscombe Garden Estates LTd. $v$. Crabbe and Others [1957] 3 WLR 980 $7,9,196$ Adler $v$. Blackman [1953] 1 QB 146 12,15 A. G. Securities $v$. Vaughan and Others (1988) 47 EG $193 \quad 8,127$ Aldin $v$. Latimer Clark Muirhead \& Co. [1894] 2 Ch 437 Aldwych Club v. Copthall Property Co. (1962) 185 EG $219 \quad 202$ American Cyanamid v. Ethicon [1975] AC 396 Anglia Building Society $v$. Sheffield City Council (1983) 266 EG 311

Antoniades $v$. Villiers and Another (1988) 3 WLR 1205

Arlesford Trading Co. Ltd. v. Servansingh [1971] 1 WLR 1080

Ashburn Anstalt $v$. W. J. Arnold \& Co. [1988] 2 WLR 706 Aslan v. Murphy (Nos. 1 \& 2) [1990] 1 WLR 766

Associated British Ports $v$. Bailey plc [1990] 2 AC 703

Barnes v. Barratt [1970] 2 WLR 1085

Barrett v. Lounova (1982) Ltd. [1989] 1 All ER 351

Bashir $v$. Commissioner of Lands [1960] AC 44

Basildon Development Corporation $v$. Mactro Ltd. (1986) I EGLR

137

78,89

Basingstoke \& Deane B. C. v. Host Group Ltd. (1988) 1 WLR 348

Bathavon R. D. C. v. Carlile [1958] 1 QB 461

Barth v. Pritchard [1990] 20 EG 65

Beacon Carpets Ltd $v$. Kirby [1984] 2 All ER 726

205

Becker $v$. Hill Street Properties [1990] 38 EG 107

Becton Dickinson UK v. Zwebner [1988] 3 WLR 1376

202

Beer $v$. Bowden (1981) 1 WLR 522

Bell v. Alfred Franks \& Bartlett Co. Ltd. [1980] 1 WLR 
Betty's Cafes Ltd. v. Phillips Furnishing Stores Ltd. [1957] Ch 67

Bickel $v$. Duke of Westminster [1977] QB 517

Billson v. Residential Apartments Ltd. [1992] AC 494

Blackburn $v$. Hussein (1988) 22 EG 78

Blatherwick (Services) Ltd. v. King (1991) 23 HLR 188

Blawdziewicz $v$. Diadon Establishment (1988) 35 EG 83

Bocardo S. A. v. S. \& M. Hotels Ltd. [1979] 3 All ER 737

Bovis Group Pension Fund Ltd. v. G. C. Flooring \& Furnishing

Ltd. (1984) 269 EG 63

Breams Property Investment Co. Ltd. v. Stroulger [1948] 2 KB 1

Brew Bros. Ltd. v. Snax (Ross) Ltd. [1970] 1 QB 612

Brickfield Properties $v$. Hughes (1987) 20 HLR 108

British Anzani (Felixstowe) Ltd. $v$. International Marine

Management (U.K.) Ltd. (1980) QB 137

British Gas Corporation $v$. Universities Superannuation Scheme

Ltd. [1986] 1 WLR 398

Brock $v$. Wollams [1949] 2 KB 388

Bromley Park Garden Estates $v$. Moss [1982] 2 All ER 890

Brooker Settled Estates Ltd. v. Ayers (1987) 19 HLR 246

Brown v. Liverpool Corporation [1969] 3 All ER 1345

Browne v. Flower [1911] 1 Ch 219

BTE Ltd. $v$. Merseyside \& Cheshire RAC ex p. Jones (1992) 16 EG

111

Buchman v. May [1978] 2 All ER 993

132

Buswell v. Goodwin [1971] 1 All ER 418

30,35

Button's Lease, $\operatorname{Re}$ [1964] Ch 263

C. \& A. Pensions Trustees Ltd. v. British Vito Investments Ltd. (1984) 272 EG 63

Cadle v. Jacmarch [1957] 2 WLR 80

Calabar (Woolwich) Ltd. $v$. Tesco Stores Ltd. (1977) 245 EG 479

Campden Hill Towers Ltd. v. Gardner and Another [1977] QB 823

Cardiothoracic Institute $v$. Shrewdcrest Ltd. [1986] 1 WLR 368

Cardshops Ltd. $v$. Davies [1971] 1 WLR 591

Carrega Properties v. Sharratt [1979] 1 WLR 928

Celsteel Ltd. v. Altan House Holdings Ltd. (No. 2) [1986] 1 All

ER 598

Central Estates (Belgravia Ltd.) v. Woolgar [1972] 1 WLR 1048150 
Centrovincial Estates Plc. v. Bulk Storage Ltd. (1983) 46 P. \& CR 393

Chamberlain $v$. Scally [1992] EGCS 90 109,115

Chapman $v$. Freeman [1978] 3 All ER 878

Cheniston Investments $v$. Waddock [1988] 46 EG 88

Cheryl Investments $v$. Saldanha, Royal Life Savings $v$. Page [1977] 1 WLR 1329

Chester $v$. Buckingham Travel Ltd. [1981] 1 All ER $386 \quad 26,37$

Church of England Building Society $v$. Piskor [1954] Ch 553 14, 17

City \& Metropolitan Properties Ltd. v. Greycroft Ltd. [1987] All ER 839

City of London $v$. Fell [1992] 3 All ER 224

Clore $v$. Theatrical Properties Ltd. [1936] 3 All ER 483

Coatsworth $v$. Johnson (1886) 54 LT 520

Cobb $v$. Lane [1952] 1 All ER 1199

Cobstone v. Maxim [1984] 3 WLR 563

Collins $v$. Hopkins [1923] 2 KB 617

Cook v. Mott (1961) 178 EG 637

205

Coronation Street Industrial Properties $v$. Ingall Industries [1989]

1 WLR 304

47,48

Crago v. Julian [1992] 1 WLR 372

Crawley Borough Council $v$. Sawyer (1987) 20 HLR 98

Cremin v. Barjack Properties Ltd. (1985) 273 EG 299

Dakyns v. Pace [1948] 1 KB 22

Denetower $v$. Toop (1991) 23 HLR 362

D'Eyncourt $v$. Gregory (1866) LR 3 Eq 382

Di Palma $v$. Victoria Square Property Co. Ltd. [1985] 3 WLR 207

Doherty $v$. Allman (1878) 3 App Cas 709

Douglas-Scott $v$. Scorgie [1984] 1 WLR 716

Dover District Council $v$. Farrar (1982) 2

Duncliffe $v$. Caerfelon Properties Ltd. [1989] 27 EG 89

Eichner $v$. Midland Bank Executor \& Trustee [1970] 1 WLR 1120

Electricity Supply Nominees Ltd. v. Thorn EMI Retail Ltd. [1991] 35 EG 114

Elite Investments Ltd. $v$. T. I. Bainbridge Silencers Ltd. (1986) 2 EGLR 43

Enfield Borough Council $v$. French (1985) 49 P\&CR 228 
Equity \& Law Life Assurance Society plc $v$. Bodfield Ltd. (1987) 281 EG 1448

Essex Plan $v$. Broadminster [1988] 43 EG 84

Essexcrest $v$. Evenlest Ltd. (1988) 285 EG 56

Expert Clothing Service \& Sales $v$. Hillgate House [1985] 2 All ER 998

Facchini $v$. Bryson [1952] 1 TLR 1386

Factors (Sundries) Ltd. v. Miller [1952] 2 All ER 630

Family Housing Association $v$. Jones [1990] 1 All ER 385

Farrell v. Alexander [1976] 3 WLR 145

Fawke $v$. Viscount Chelsea [1979] 3 All ER 568

Finchbourne $v$. Rodriguez [1976] 3 All ER 581

Fuller $v$. Judy Properties Ltd. [1992] 14 EG 106

Gardner \& Co. Ltd. v. Cone [1928] Ch 955

Gladstone v. Bower [1960] 3 WLR 575

Glass $v$. Kencakes Ltd. [1966] 1 QB 611

Goodrich v. Paisner [1956] 2 WLR 1053

Gordon $v$. Selico Ltd. [1986] 1 EGLR 71 EG 106

Grand Junction Co. Ltd. v. Bates [1954] 2 QB 160

Greenwich L.B.C. v. McGrady (1983) 46 P \& CR 223

Griffith $v$. Pelton [1958] Ch 205

Gurton v. Parrott [1991] 18 EG 161

Hagee (London) Ltd. v. A. B. Erikson \& Larson [1976] QB 209195 Haines $v$. Florensa (1990) 59 P \& CR 200

Halliard Property Co. Ltd. v. Nicholas Clarke Investments Ltd. (1984) 269 EG 1257

Hammersmith \& Fulham L.B.C. v. Monk [1991] 3 WLR $1144 \quad 115$

Hampshire v. Wickens (1878) 7 Ch D 555

Hamstead \& Suburban Properties Ltd. v. Diomedous [1969] 1 Ch 248

Hampstead Way Investments $v$. Lewis-Weare [1985] 1 WLR 164

Harewood Hotels $v$. Harris [1958] 1 WLR 108

Harris $v$. Swick Properties [1969] 1 WLR 1604

170

Harrogate Borough Council v. Simpson (1984) 17 HLR 205

Hart v. Emelkirk [1983] 3 All ER 15

Haskell $v$. Marlow [1928] 2 KB 45

Heath $v$. Drown [1972] 2 WLR 1306 


\section{xiv Table of Cases}

Herongrove Ltd. $v$. Wates City of London Properties (1988) 24 EG 108

Heslop v. Burns [1974] 3 All ER 406

Hibernian Property Co. v. Liverpool Corporation [1973] 1 WLR 751

Hill $v$. Barclay (1810) 16 Ves 402

Hill $v$. Rochard [1983] 1 WLR 98

Hillbank Properties Ltd. v. Hackney London Borough Council [1978] QB 998

Hillil Property and Investment $v$. Naraine Pharmacy (1979) 252 EG 1013

Hopwood v. Cannock Chase D.C. [1975] 1 WLR 373

Hogarth Health Club v. Westbourne Investments [1990] 62 EG 69

Houlder Bros. \& Co. v. Gibbs [1925] Ch 575

209

Hussein v. Mehlman [1992] 52 EG 39

Hyde Housing Assoc. v. Harrison (1991) 23 HLR 57

Hyman $v$. Rose [1912] AC 623

International Drilling Fluids Ltd. $v$. Louisville Investments

(Uxbridge) Ltd. [1986] 1 All ER 321

Irvine $v$. Moran [1991] 1 EGLR 261

Jefferies $v$. O'Neill (1983) 269 EG 131

69

Jeune v. Queens Cross Properties Ltd. [1974] Ch 97

61

Johnsey Estates $v$. Webb [1990] 19 EG 84

Kay Sandra McNerny $v$. L. B. Lambeth (1988) 21 HLR 188

Kaye $v$. Massbitten \& Kaster (1992) 24 HLR 28 127

Kenneally $v$. Dunne [1977] 2 WLR 421

Kenny $v$. Preen [1963] 1 QB 499

Killick and Another $v$. Second Covent Garden Property Co. Ltd. [1973] 1 WLR 658

King $v$. South Northamptonshire DC (1991) EGCS 114

Lace $v$. Chantler [1944] KB 368

Ladup Ltd. v. Williams \& Glyns Bank plc [1985] 2 All ER $577 \quad 116$

Lambert $v$. F. W.Woolworth \& Co. Ltd. (No. 2) [1938] Ch 883 80, 81 Land Reclamation Co. Ltd. v. Basildon D.C. [1979] 2 All ER 993

Law Land Co. Ltd. v. Consumers' Association Ltd. (1980) 255 EG 617 
L. B. Greenwich $v$. Powell (1989) 21 HLR 168

L. B. Hammersmith \& Fulham v. Monk (1991) 23 HLR 1114

L. B. Waltham Forest $v$. Thomas [1992] 3 All ER 244

Lear $v$. Blizzard [1983] 3 All ER $662 \quad 83,91$

Lee $v$. K. Carter Ltd. [1949] 1 KB 85

Lee-Parker $v$. Izzet [1971] 1 WLR 1688

Lee-Verhulst $v$. Harwood Trust (1978) 250 EG $971 \quad 195,196$

Lees $v$. Tatchell [1990] 23 EG $62 \quad 217$

Leigh $v$. Taylor (1902) AC $157 \quad 117$

Lister v. Lane [1893] 2 QB 212

Liverpool City Council $v$. Irwin [1977] AC 239

London \& Associated Investment Trust PLC v. Calow (1986)

280 EG 1252

194

London Housing and Commercial Properties Ltd $v$. Cowan [1977] QB 148

Loria $v$. Hammer [1989] EG CS 126

Lower $v$. Sorrell [1963] 1 QB 959 108

Luganda $v$. Service Hotels Ltd. [1969] 2 Ch. $209 \quad 136,179$

Lurcott $v$. Wakely \& Wheeler [1911] 1 KB 905

Lynnthorpe Enterprises Ltd. $v$. Sidney Smith (Chelsea) Ltd. (1990) 2 EGLR 131

McAuley v. Bristol City Council (1992) 1 QB $434 \quad 33,85$

McDonnel $v$. Daly [1969] 1 WLR 1482

McDougall v. Easington D.C. (1989) 58 P \& CR 201

McGreal $v$. Wake (1984) 269 EG 1254

Malpas v. St Ermin's Property Co. Ltd. [1992] 24 HLR $537 \quad 169$

Mammoth Greeting Cards Ltd. v. Agra Ltd. [1990] 29 EG $45 \quad 86$

Mancetter Developments Ltd. v. Garmanson Ltd. [1986] 2 WLR 871

Mander $v$. Falcke [1891] 2 Ch $554 \quad 50,52$

Manfield \& Sons Ltd. v. Botchin (1970) 3 WLR $120 \quad 14,15$

Marcroft Wagons Ltd. v. Smith [1951] 2 All ER 271

Marjorie Burnett Ltd. v. Barclay (1980) 258 EG 642

Mars Security Ltd. v. O'Brien [1991] 2 EGLR 281 87

Metropolitan Properties v. Finegold [1975] 1 WLR $349 \quad 138$

Metropolitan Properties Co. Ltd. v. Cordery (1979) 39 P \& CR 10

116

Midland Bank Trust Co. Ltd. v. Green [1981] AC 513

Midland Railway Company's Agreement, Re [1971] 1 All ER 1007

Mikeover $v$. Brady [1989] 3 All ER 618

Mint $v$. Good [1951] 1 KB 517 
Mira $v$. Aylmer Savaro Investments (1990) 22 HLR 182

Mirror Group (Holdings) Ltd. In re (1992) 63 P \& CR 252

Mistry $v$. Isidore [1990] 31 EG $43 \quad 152$

Moat $v$. Martin [1950] 1 KB 175

Moule v. Garrett (1870-72) LR 5EX $132 \quad 48,49$

Mumford Hotels Ltd. v. Wheler [1964] Ch $117 \quad 81,90$

Mykolyshyn $v$. Noah [1970] 1 WLR 1271

Narcissi $v$. Wolfe [1959] 3 WLR 431

National Carriers Ltd. v. Panalpina (Northern) Ltd. [1981] AC $675 \quad 66,86,113$

Nelson Developments Ltd. $v$. Taboada (1992) 24 HLR 462

Newman $v$. Dorrington Developments Ltd. [1975] 3 All ER $928 \quad 83$

New Zealand Government Property Corporation v. H. M. \& S.

Ltd. [1982] 1 All ER 624

O'Brien $v$. Robinson [1973] AC 912

Official Custodian for Charities $v$. Parway Estates Developments Ltd [1984] 3 All ER 679

O'Leary $v$. London Borough of Islington [1983] 9 HLR 81

O'May $v$. City of London Real Property Co. Ltd. [1983] 2 AC 72

Otter $v$. Norman (1989) 20 HLR 594

Owen $v$. Gadd [1956] 2 QB 99

P. \& A. Swift Investments $v$. Combined English Stores Group PLC [1988] 2 WLR 313

Palmer $v$. Sandwell Metropolitan Council (1987) 20 HLR $74 \quad 182$

Palmer $v$. McNamara (1991) 23 HLR $168 \quad 130$

Palser v. Grinling [1948] AC 291

Parker $v$. Camden London Borough Council [1985] 2 All ER 14162

Patman v. Harland (1881) 17 Ch D $353 \quad 105$

P. F. Aher \& Sons Ltd. v. Hunt (1988) 21 EG 69

Pitcher $v$. Tovey (1692) 4 Mod $71 \quad 86$

Plinth Property Investments Ltd. v. Mott, May \& Anderson (1979) P \& CR 361

Podbury $v$. York (1990) 41 EG 65

Poland v. Earl Cadogan (1980) 250 EG $495 \quad 170$

Ponsford $v$. H.M.S. Aerosols Ltd. [1979] AC 63

Post Office Counters Ltd. v. Harlow D.C. [1991] 2 EGLR 121

Premier Confectionery (London) Co. Ltd. v. London Commercial Sale Rooms Ltd. [1933] Ch 904

Prudential Assurance Co. v. London Residuary Body [1992] 3 WLR 279 
Proudfoot $v$. Hart (1890) 25 QBD 42

Pulleng $v$. Curran (1980) 44 P \& CR 58

Purchase $v$. Lichfield Brewery Co. [1915] 1 KB 184

Queensway Marketing Ltd. v. Associated Restaurants Ltd. (1984) 271 EG 1106

Quick v. Taff-Ely B.C. [1986] QB 809

R. v. Burke (1990) 22 HLR 433

R. v. L. B. Hillingdon, ex parte Pulhoffer [1986] 2 WLR 259

R. v. London Rent Assessment Panel, ex parte Trustees of Henry Smith's Charity (1988) 20 HLR 103

Ravenseft Properties Ltd. v. Davstone (Holdings) Ltd. [1980] QB 12

Redspring $v$. Francis [1973] 1 WLR 134

Regis Property Co. Ltd. v. Dudley [1959] AC 370

Reohorn $v$. Barry Corporation [1956] 1 WLR 845 43,58

Robert Thackray's Estate v. Kaye (1989) 21 HLR 160

Ross Auto Wash $v$. Herbert (1978) 250 EG 971

130

Ross $v$. Collins [1964] 1 WLR 425

196

Rugby School $v$. Tannahill [1935] 1 KB 87

137

Rutherford $v$. Maurer [1961] 3 WLR 5

116

Rye $v$. Rye [1962] AC 496

Sanderson $v$. Berwick on Tweed Corporation (1884) 13 QBD 547

Saner $v$. Bilton (1878) 7 Ch D 815

Saunders $v$. Soper [1974] 3 WLR 777

Scala House \& District Property Co. Ltd. v. Forbes [1974] QB 575

Sharpe $v$. Duke Street Securities (1987) 19 HLR 506

Shaw $v$. Groom [1970] 2 WLR 299

Sheldon $v$. West Bromwich Corporation (1973) 117 Sol J 486

32,85

Siddiqui $v$. Rashid [1980] 1 WLR 1081

158

Skeet $v$. Powell-Sheddon [1988] 40 EG 116

207

Smiley $v$. Townshend (1950) 2 KB 311

Smith's Lease, $\operatorname{Re}(1951) 1$ All ER 346

Smith $v$. Bradford Metropolitan Council (1982) 44 P \& CR 171

Smith $v$. Marrable (1843) 11 M \& W 5

Solomon $v$. Orwell [1954] 1 All ER 874

S. Northants D.C. v. Power (1987) 20 HLR 133 
Spencer's Case (1583) 5 Co Rep 16a 47, 83

Spook Erection Ltd. $v$. British Railways Board (1988) 21 EG 73205

Spraggs $v$. Prentice (1950) 100 LJ 541

Strand \& Savoy Properties Ltd., Re [1960] Ch 582

Strand Securities Ltd. v. Caswell [1965] Ch 958

Street $v$. Mountford [1985] AC 809

Sudbrook Trading Estate Ltd. v. Eggleton [1983] 1 AC $444 \quad 68,84$

Tagro v. Cafane (1991) 2 All ER 235

Tandon $v$. Spurgeon's Homes [1982] 2 WLR 785

Torbett $v$. Faulkener [1952] 2 TLR 659

Torrens $v$. Walker [1906] 2 Ch 166

Town Investments Ltd. Underlease, $\operatorname{Re}[1954]$ Ch 301

Trustees of Henry Smith's Charity $v$. A.W.A.D.A. Trading \& Promotion Services Ltd. (1984) 47 P \& CR 607

United Scientific Holdings $v$. Burnley Borough Council [1978] AC 904

Vural Ltd. v. Security Archives (1990) 60 P \& CR 258

Wansbeck D.C. v. Marley [1988] 20 HLR 247

Ward $v$. Warnke (1990) 22 HLR 496

Warmington $v$. Miller [1973] QB 877

Warren $v$. Keen [1954] 1 QB $15 \quad 42,43$

Webb v. Barnet L.B. [1989] 11 EG 80

Webb v. Pollmount Ltd. [1966] Ch $584 \quad 51,90$

West Country Cleaners Ltd. v. Saly [1966] 1 WLR 1485

Westminster City Council v. Clarke 1992 2 WLR 229

White v. Bijou Mansions Ltd. [1937] Ch $610 \quad 105$

Whiteminster Estates $v$. Hodges Menswear (1974) 232 EG $715 \quad 76$

Wilson $v$. Flynn [1948] 2 All ER $40 \quad 88$

Woolff $v$. Waddington [1989] 47 EG 148

Woolworth (F.W.) \& Co. Ltd. v. Charlwood Alliance Properties Ltd. (1987) 282 EG 585

Wycombe Health Authority v. Barnett (1982) 264 EG $619 \quad 32,43$ 


\section{Table of Statutes}

Accommodation Agencies Act 1953 s.1

Administration of Justice Act 1985 s.55

Agricultural Holdings Act 1947 s.10

Agricultural Holdings Act 1986

s. 2

213

s.2(1)

213

s.2(2)

213

s.2(3)

213

s. 3

213

s. 5

213

s. 6

214

s.12

215

s.15

215

s. 22

214

s. 25

216

s. 26

216

s. 27

216

s.31

217

s. 60

219

s. 67

219

s.79

219

s. 80

219

s.96

213

Schedule 1

Schedule 2

215

Schedule 3

Schedule 7

Schedule 8

Agriculture (Maintenance, Repairs and Insurance of

Fixed Equipment Regulations) 1973

Allotments Acts 1922-50 
xx Table of Statutes

Common Law Procedure Act 1852

Conveyancing Act 1881, s.14(1)

County Courts Act 1984, s.138

Criminal Law Act 1977, s.6(1)

Defective Premises Act 1972

s. 1

33

s. 4

$33,38,59$

Environmental Protection Act 1990

65

Housing Act 1985

178

s. 21

s. 24

184

s.79

s.79(3)

184

s.79(4)

$126,179,187$

s.80

178

178

s.81

179

s.83

129, 179

s.87

185

s. 89

183

s.91

182

s.92

182

s.93

181

s.94

181

181

s.94(5)

181

s.96

182

ss. 97-101

182

ss. 102-103

182

ss.104-106

182

s.113

184

s.189

63

s. 190

64

s.193

64

s. 264

64

s.604

64

Part III

177

Part IV

178

Part V

183

Part VII

Schedule 1

180

Schedule 2 
Table of Statutes $\quad \mathrm{xxi}$

Housing Act 1988

128,176

s.1(1)

s.1(3)

$126,129,167,197$

s.3(5)

140

s.5(4)

128

s.5(5)

141

s. 6

141

s.7

141,142

s.7(6)

143

s.8

143

s.8(1)

143

143

s.8(3)

143

s.8(5)

143

s. 9

s.9(3)

143,149

143

s. 10

143

s.12

144

s. 13

141

s.13(2)

142,145

s.14

s.15(1)

141,142

142

143

s.15(2)

143

143

s.15(4)

143

144

s.17

136, 141

144

144

145

145

145

145

s. 23

220

ss. 24-26

147

148

s.27(1)

147

147

s. 28

147

s. $28(3)$

148

s. 30

148

s. 31

s. 34

134, 168

s.34(2)

135 
xxii Table of Statutes

s.35

179

s. 37

s.38

179

s. 39

135

s. 44

132

s. 115

140

s.116

31,37

Schedule 1

$129,130,131$

Schedule 2

$143,149,154-156,157,187$

Land Charges Act 1925

98,99

Land Charges Act 1972

$50,98,101$

Land Compensation Act 1973

177,209

Land Registration Act 1925

98

s.8(2)

105

s.70(1)(g)

s.70(1)(k)

$47,83,84,100,105$

84,100

s.77

105

Land Registration Act 1986

s. 1

100,105

s. 3

105

191

208

s. 17

59

s.18(1)

s.19(1)

s.19(2)

s.19(3)

s. 47

$74,75,87,109,208$
$80,81,90$

$78,79,89$

209

Landlord \& Tenant Act 1954

s. 3

168

s. 4

168

s. 10

169

s. 23

191, 196, 197

s.23(1)

194, 206

s.23(2)

196

s.24(1)

198,200

s.24(2)

197

s. $24 \mathrm{~A}$

200,201

s. $24 \mathrm{~A}(1)$

201

201

s. $24 \mathrm{~A}(3)$

$199,200,203,207$
200

s. 25

199, 200, 203, 207

s.25(5)

s. 26

$199,200,203,207$ 
Table of Statutes $\quad \mathrm{xxiii}$

s. $26(6)$

$$
\text { s. } 27
$$

s.29(2)

s.29(3)

s.30

s.30(1)

s.30(2)

s.31

s.31(2)

s.31A

s.32

s.32(1A)

s. 33

s. 34

s.34(3)

s. 35

s.36(1)

s.36(2)

s. 37

s. 38

s. $40(1)$

s. $40(2)$

s. 41

s. $41 \mathrm{~A}$

s. 42

s. $43(1)(a)$

s.43(1)(b)

s. $43(2)$

s.43(3)

s. 44

s. 47

s.55

s.57(3)

s. 64

s. 65

Part I

Part II

Landlord \& Tenant Act 1985

s. 4

s. 5

s. 7

s. 8

s. 11

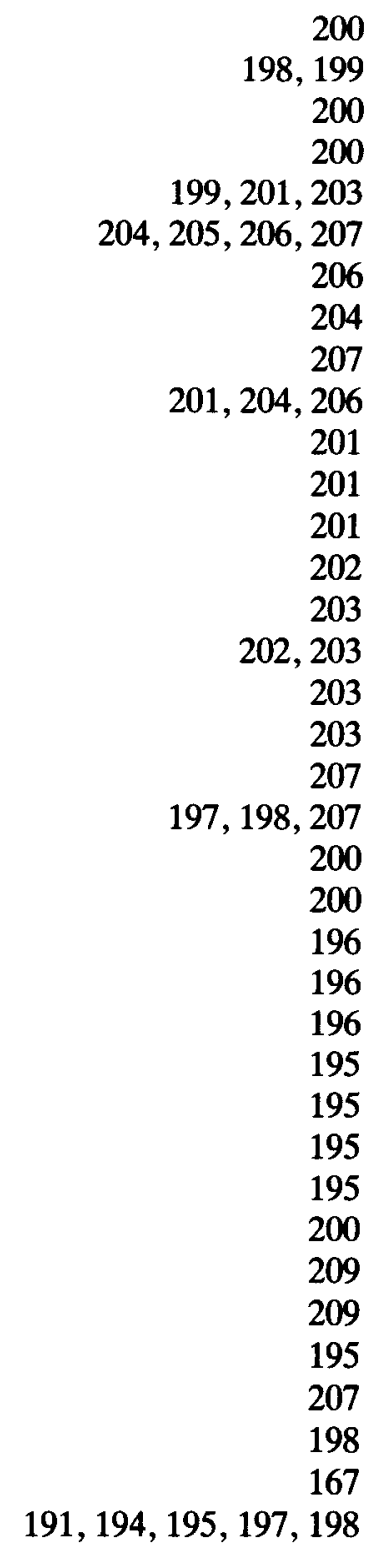

$41,66,145,180$

180

146

30

$113,181,182$ 
xxiv Table of Statutes

s.11(1)

$30,31,34,36,43,55$

s.11(3)

32

s.11(6)

43

s.12(2)

33

s.17

61

s. 18

163

s.19

163

s. 20

163

s. $20 \mathrm{~B}$

164

s. 21

163

s. 29

164

s.30B

164

s. 47

164

Landlord \& Tenant Act 1987

162

s. 2

165

s. 3

165

s. 4

165

s. 24

166

s. 25

167

s. 29

166

s.35

167

s. 38

167

s. 60

$32,38,165$

Part I

165

Part II

166

Part III

62,166

Part IV

167

Landlord \& Tenant Act 1988

77,78

s.1(3)

s.1(5)

78

s. 4

89

78

195

Landlord \& Tenant (Licensed Premises) Act 1990

71

50

s.6

13,46

Law of Property Act 1922

Law of Property Act 1925

s.1(1)

19

s.40

19,21

s. $44(2)$

105

$\mathrm{s.44(3)}$

105

$\mathrm{s.44(5)}$

100

s.52

20

$\mathrm{s.54(2)}$

$11,20,22,41,47,98,100$ 


\section{Law of Property Act 1969}

Law of Property (Miscellaneous Provisions)

\section{Act 1989}

\section{s. 2 \\ s.2(5)(a) s.2(8)}

Leasehold Property (Repairs) Act 1938

Leasehold Reform Act 1967

s. 1

s. 2

s. 3

s. 4

s.4A

s.7

ss. 8-13

ss. 14-16

s. 16

s.17

s. 18

s. 28

Schedule 3
20,26

20

21 $59,60,61$ 169

169 169 169 169

169 170 170 170 195 170 170 170 170

Leasehold Reform Housing \& Urban Development Act 1993 
$\begin{array}{ll}\text { s.1(7) } & 173\end{array}$

s. 2

173

s.3

171

s.3(1)

172

s. 4

171

s.4(4)

171

s.5

172

s.7

172

s. 8

172

s. 10

171

s.11

172

s. 13

172

s. 15

172

s. 17

172

s. 23

173

s. 26

172

s. 32

172

s. 39

174

s. 47

174

s.56

174

s. 57

174

s. 61

174

ss.69-75

173

Schedule 5

172

Schedule 12

174

Limitation Act 1980

5,12

Schedule I Part I

15

Local Government \& Housing Act 1989

Schedule 4

129,168

208

Mobile Homes Act 1983

127

Notice to Quit (Prescribed Information) Regulations 1975

148

Perpetuities \& Accumulations Act 1964, s.9(1)

84

Protection from Eviction Act 1977

131,132

s.1

29

s.1(2)

147

s.1(3)

$28,146,147$

s.1(3A)

146,147

s. 3

148

s.3A 
Table of Statutes xxvii

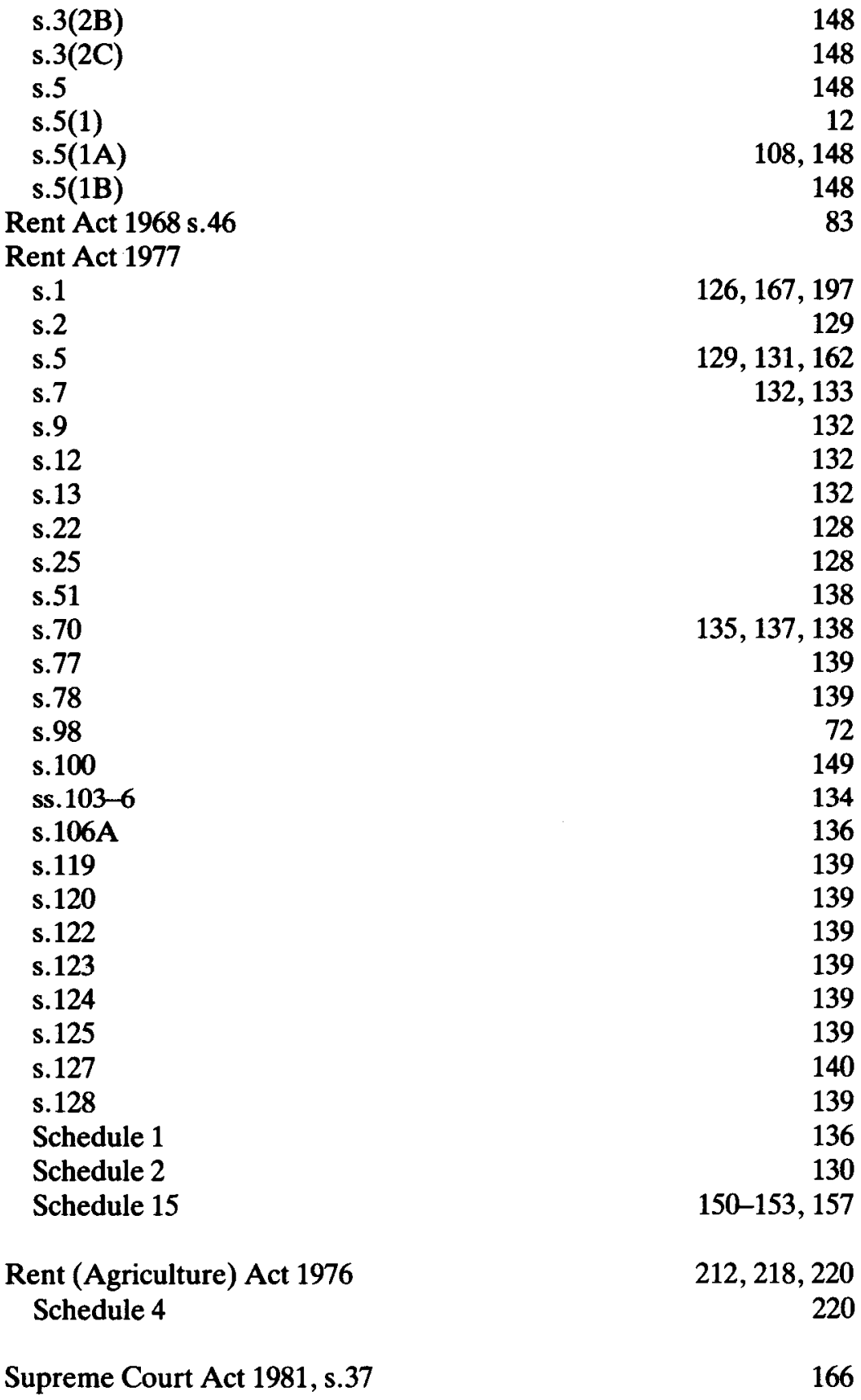




\section{Preface}

This book is written primarily as a student text and is designed in particular to be a self-teaching aid. To this end, there are summaries, exercises and workshops at the end of each chapter. The aim of the book is to give the student a sound basic knowledge of the law of landlord and tenant, a subject which is included in many professional courses.

The first part of the book deals with the nature of a lease in the context of land law and the basic obligations of the parties to it. It examines the position on the disposition of a lease, the validity of a lease against third parties and how a contractual lease is terminated. It also includes a chapter on the conveyancing aspects of a lease in unregistered and registered title and a short form of model lease.

The second part examines the impact of statute on leases. Residential, business and agricultural leases are all subject to detailed and complex legislation affecting one or more of security of tenure, terms and rent and control.

The second edition now includes recent cases of substantial import in various areas of the basic law, notably on repairs, rent review, liability on covenants and the House of Lords decision on relief against forfeiture under s.146 LPA. It has presented the opportunity too to explore the ramifications of recent legislation such as the Housing Act 1988, not to mention the implications of new legislation, notably the Leasehold Reform Housing and Urban Development Act 1993.

Finally, the need for a new edition just four years after the first is illustrative of the speed, significance and impact of changes in case law as well as procedure in this area of the law.

Margaret Wilkie GODFREy COLE 